\title{
Dental Care in the Arab Countries During the COVID-19 Pandemic: An Infodemiological Study
}

\author{
Khalifa S Al-Khalifa $\mathbb{D}^{\prime}$ \\ Rasha AISheikh (D) ${ }^{2}$ \\ Yaser A Alsahafi ID $^{3}$ \\ Atheer Alkhalifa ${ }^{4}$ \\ Shazia Sadaf $\mathbb{D}^{5}$ \\ Saud A Al-Moumen ${ }^{6}$ \\ Yasmeen $Y$ Muazen $^{7}$ \\ Ashwin C Shetty ${ }^{8}$ \\ 'Department of Preventive Dental \\ Sciences, College of Dentistry, Imam \\ Abdulrahman Bin Faisal University, \\ Dammam, Saudi Arabia; ${ }^{2}$ Department of \\ Restorative Dental Sciences, College of \\ Dentistry, Imam Abdulrahman Bin Faisal \\ University, Dammam, Saudi Arabia; \\ ${ }^{3}$ Department of Preventive Dental \\ Sciences, College of Dentistry, Taibah \\ University, Al-Madinah Al-Munawwarah, \\ Saudi Arabia; ${ }^{4}$ The National Center for \\ Artificial Intelligence and Big Data, King \\ Abdulaziz City for Science and \\ Technology (KACST), Riyadh, Saudi \\ Arabia; ${ }^{5}$ Department of Dental \\ Education, College of Dentistry, Imam \\ Abdulrahman Bin Faisal University, \\ Dammam, Saudi Arabia; ${ }^{6}$ Dental \\ Department, Ministry of Health, \\ Dammam, Saudi Arabia; ${ }^{7}$ Dental \\ Internship Program, College of Dentistry, \\ Imam Abdulrahman Bin Faisal University, \\ Dammam, Saudi Arabia; ${ }^{8}$ Vice Deanship \\ for Postgraduate Studies and Scientific \\ Research, College of Dentistry, Imam \\ Abdulrahman Bin Faisal University, \\ Dammam, Saudi Arabia
}

Correspondence: Khalifa S Al-Khalifa Department of Preventive Dental Sciences, College of Dentistry, Imam Abdulrahman Bin Faisal University, Dammam, Saudi Arabia

Email kalkhalifa@iau.edu.sa
Background: Twitter is a powerful platform which could be used to reflect on the demand and supply of dental services during a pandemic. The aim of this study was to examine the nature and dissemination of COVID-19 information related to dentistry on Twitter platform Arabic database during the COVID-19 pandemic.

Methodology: One hundred and fifty independent searches with a combination of keywords for both COVID-19 and dentistry from a preselected Arabic keyword were carried out for the period from the 2nd of March (first confirmed cases of COVID-19) to the 6th of July 2020. Tweets were filtered to remove duplicate and unrelated tweets. The suitable tweets were 1,150. After calibration, two examiners coded the tweets following two main themes: COVID-19 and oral health-related information. Tweets were then compared with COVID19 daily events in the Arab countries as reported by the World Health Organization (WHO). Descriptive analysis was performed to present the overview of the findings using Microsoft Excel.

Results: The most retweeted information was the help with urgent consultation or emergency dental treatment during COVID-19 tweeted by a dentist. There were 673 retweets and 1,116 likes of this tweet. The most common tweets related to oral health were needs of dental treatment $(n=462,39.5 \%)$ of which, toothaches or wisdom tooth problems constituted $48 \%$ of the related tweets.

Conclusion: Based on the results of this study, it is obvious that social media users reacted to the COVID-19 threat to dental practices. Twitter as one of the social media platforms served as a connection between dental health professionals and patients.

Keywords: COVID-19, Twitter, pandemic, oral health, dentistry, social media, tweet, Arab

\section{Introduction}

Coronavirus (COVID-19) is a worldwide pandemic, which started as a severe acute respiratory disease first called coronavirus 2 (SARS-CoV-2). COVID-19 as an epidemic first started in Wuhan, China and then spread rapidly around the globe, with a rise in number of infections and associated deaths' numbers. ${ }^{1,2}$ As of 8 October 2020, and since the outbreak of the COVD-19 (31 December 2019), there have been 36,194,764 cases and 1,053,961 deaths from more than 200 countries worldwide. ${ }^{3}$ The symptoms of this disease range from dry cough and fever to other symptoms such as sore throat, shortness of breath, diarrhea, muscular pain, fatigue, vomiting, and conjunctivitis. ${ }^{4}$

As seen in numerous countries, Saudi Arabia and other Arab countries began taking precautionary actions preventing sudden and sharp increase in the number of cases. Despite active prevention and surveillance programs, inducted by ministries 
of health in Arab countries to better control the spread of the pandemic, COVID-19 cases have been increasing steadily. As of 1 st of October 2020, there was 2,559,068 reported total cases, 2,194,948 cured/recovered and 65 , 356 deaths in Arab states. ${ }^{5}$

The transmission mode of COVD-19 among healthcare providers has been documented, where dental professionals are considered at high risk for non-social infection. ${ }^{6}$ The threats with the identification of COVID19 positive patients or carriers among dental patients are due to various aerosols-generating procedures involved in dental care, lengthy incubation period, and delayed symptom onset. Thus, as a precautionary measure during this pandemic, the majority of dental practices were either suspended for all treatments or were open partially to handle the emergency. ${ }^{1,7,8}$ Ethically, dentists are responsible to provide emergency dental care for patients regardless of their health status, and to balance between the patients' needs and public health concerns. ${ }^{9,10}$

Many health care professional and governing bodies have utilized social media platforms for spreading the awareness about COVID-19 pandemic. ${ }^{11}$ Dentistry and dental health-care professionals were not exceptional to this, which lead to increased use of social media during the pandemic for exchanging information about dentistry and oral health with the public. Social media has a significant conduit in the modern media environment for sharing news and information. The pattern of spread of news in social media made a positive change for the way health-care providers and patients communicate on health-related information. ${ }^{1}$

Social media users could identify the main attitudes, feelings, thoughts, and topics that have expressions related to the COVID-19 pandemic. ${ }^{12}$ One such big platform utilized commonly to search information and exchange thoughts among other uses is Twitter. Twitter is a microblogging platform for users to share their thoughts, questions, pictures, and links through brief online messages better known as tweets. ${ }^{13}$ Twitter platform had a significant role in the COVID-19 pandemic communications. Millions of tweets related to the virus are being posted daily. This platform is considered as a medium for the spread and consumption of news, including news about the pandemic, and capturing the dynamics of real-world events. ${ }^{14}$ Therefore, 59\% of the Twitter users describe it as good or extremely good platform for sharing preventive health information. ${ }^{15}$
Arabic is considered the official language of more than 22 countries with nearly 300 million native speakers worldwide. ${ }^{16}$ Furthermore, Twitter has a large daily availability of Arabic content, with millions of Arabic users using the social media for communication. ${ }^{17}$ For instance, Saudi Arabia had approximately 15 million Twitter users as of January 2020. Hence, analyzing the Arabic users' behavior and sentiment during this pandemic is very important. ${ }^{18}$

Analyzing the tweets about this event offers a great opportunity in understanding the structure, characteristics and development of the disease. Since the report of the first COVID-19 case in China, the pandemic had drawn the attention of a lot of Arab users in Twitter platform. Arab users' interest had increased with this ongoing pandemic on Twitter, which covered topics such as; disease general information, precautionary measures, daily pandemic statistics, new enforced decisions of the governments and change in daily habits and work routines. ${ }^{19}$

Thus, the aim of this study was to examine the nature and dissemination of COVID-19 information related to dentistry and oral health information on Twitter platform Arabic database and provide a summary on the supply and demand of dental services during the COVID-19 pandemic on Twitter social media platform.

\section{Methods}

\section{Search Strategy}

A study of "COVID-19-related oral health information" tweets on twitter in Arabic language was performed similar to the methodology of a previous Chinese study. ${ }^{1}$ Variations of keywords related to COVID-19 (coronavirus, COVID-19, epidemic, pandemic, quarantine, and lockdown) and keywords for dentistry (dentist, dentistry, and teeth) in Arabic were used to search COVID-19 and dentistry-related tweets on Twitter. The following is a list of keywords used in this study:

COVID-19 terms: Social Distancing, Prohibition, Lockdown, Pandemic, Epidemic, COVID-19, COVID 19,

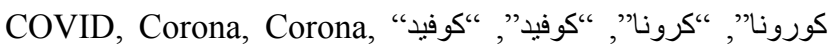

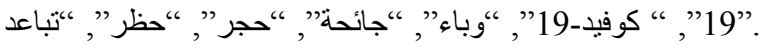

Dental terms: Dentistry (different spellings), Mouth,

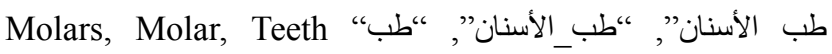

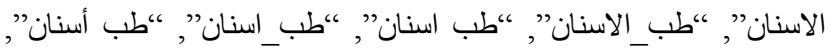

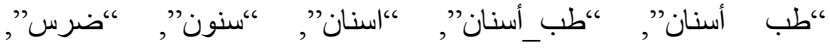

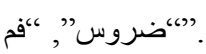

One hundred and fifty independent searches with a combination of keywords for both COVID-19 and 
dentistry were carried out on July 6, 2020. We selected March 2nd, 2020, as the start date of tweets since, on this day, the Ministry of Health confirmed the first case in Saudi Arabia. All Twitter data, including full-text content; in-text hashtags; mentions; numbers of likes, comments, and shares; post time of each tweet were extracted with a Python-based library GetOldTweets3, this program retrieved all the tweets from each independent search for this study. ${ }^{20}$

\section{Coding}

The coding was performed by two researchers (YM and SM) with experience in dentistry. Both coders conducted a pilot by coding $200(17.4 \%)$ of the total 1,150 tweets. Two main themes were identified: COVID-19 and oral health-related information. The second theme "oral healthrelated information" was further sub-classified into three domains; Dental services during COVID-19, Needs of dental treatment, and Home oral care information. Dental services during COVID-19 pandemic domain were subclassified to COVID-19 risk during dental procedures, Partial or complete closure of dental services during the pandemic, Protective measures to cut off/decrease COVID-19 transmission in dental practices and Notices of restoring dental services. While home oral care information domain was subclassified to Daily oral care tips, including some commercial ads for oral hygiene products, Management of Dental emergencies at home and Online consultation services provided by dentists, dental clinics/ hospitals, online health platforms, etc. (Table 1)

A weighted Kappa was utilized to test the reliability. Both researchers classified another 200 randomly selected tweets and reclassified one week after the first coding. The weighted Kappa was 0.882 for inter-agreement and 0.975 (YM) and 0.892 (SM) for intra-agreement with an overall excellent reliability. All 1,150 tweets were classified by one researcher and revised by the other researcher.

\section{Analysis}

Time distribution analysis was conducted by counting the total number of tweets for the designated period (2nd of March to 6th of July 2020) and comparing it with COVID19 daily statistics of new cases and deaths in the Arab countries. The epidemiological data for COVID-19 was obtained from the World Health Organization (WHO) official website 21 Oral health-related tweets were further analyzed. This included tweets on the risks of COVID-19 transmission during dental procedures, news on partial or complete closure of dental services, home oral care, need for dental treatment, COVID-19 protective measures during dental services, and news of restoring dental services during the COVID-19 pandemic. Two subclassifications were further categorized and counted, namely Needs for dental treatment and Home oral care information.

Statistical analysis for this study was carried out using Microsoft Excel (Microsoft Corporation). Descriptive analysis was performed to present the overview of the findings. This included the top five disseminated information frequently retweeted and liked on Twitter related to the study as well as needs for dental treatment during COVID19 as reported by Arab Twitter users. The results were presented in the form of frequency tables with percentages as well as graphical presentation in the form of bar charts and line charts.

\section{Results}

In total, 15,701 tweets of the COVID-19 epidemic were identified. Figure 1 shows the flow chart used in this study. A total of 7,820 tweets were scanned after removing the duplicates and 5,205 tweets were included for analysis after screening for further eligibility assessment. The remaining 1,150 tweets were finally included for further analysis. Two researchers (YM and SM) screened and assessed eligibility of the tweets.

Among 1,150 oral health-related information tweets included, the most common tweets related to oral health were needs of dental treatment $(n=462,39.5 \%)$, followed by dental service-related information during COVID-19 pandemic $(\mathrm{n}=296,25.3 \%)$, information about the prevention $(\mathrm{n}=257, \mathrm{n}=22.0 \%)$, and home oral care information $(n=155,13.2 \%)$. Of the 155-home oral care information tweets, information about dental advise $(\mathrm{n}=89,57.4 \%)$ witnessed the most tweets as well as information about the daily oral care $(n=59,38.1 \%)$ and the remainder were tweets about dental consultation $(\mathrm{n}=7,4.5 \%)$.

Figures 2 and 3 show the time distribution of tweets to the distribution of COVID-19 new cases and deaths between March 2, 2020 and July 6, 2020. The daily count of tweets increased with fluctuations starting on March 3, 2020, this was followed by three major peaks before May 1, 2020, and kept a steady low until July 6, 2020. On the other hand, the daily number of new cases of COVID-19 was low during the first two weeks of March, then increased with fluctuations starting mid-March, 2020, presented one major drop on May 22, 2020, and kept a steady increase until 
Table I Classification of COVID-19 and Dentistry/Oral Health-Related Tweets

\begin{tabular}{|c|c|c|}
\hline $\begin{array}{l}\text { Thematic } \\
\text { Categories }\end{array}$ & Subclassification & Example \\
\hline \multicolumn{3}{|c|}{ I. COVID-19 related information } \\
\hline COVID-19 Prevention & $\begin{array}{l}\text { Measures to protect individuals from COVID-19, including wearing } \\
\text { masks, washing hands, social distancing, and even some incorrect } \\
\text { methods and refutations for the misinformation, etc. }\end{array}$ & $\begin{array}{l}\text { The preventive guideline booklet of (COVID- } \\
\text { 19) } \\
\text { @mohe_sa @kauweb @KAU_FD @kauhpI }\end{array}$ \\
\hline \multicolumn{3}{|c|}{ II. Oral health related information } \\
\hline \multirow{4}{*}{$\begin{array}{l}\text { Dental services during } \\
\text { COVID-19 pandemic } \\
\text { domain }\end{array}$} & - COVID-19 risk during dental procedures & $\begin{array}{l}\text { WHO: dentistry is most vulnerable to } \\
\text { Corona infection }\end{array}$ \\
\hline & - Partial or complete closure of dental services during the pandemic & $\begin{array}{l}\text { (ADA) recommends delaying non-emergency } \\
\text { dental treatments for three weeks due to } \\
\text { Corona } \\
\text { \#COVID2019 }\end{array}$ \\
\hline & $\begin{array}{l}\text { - Protective measures to cut off/decrease COVID-19 transmission in } \\
\text { dental practices. }\end{array}$ & $\begin{array}{l}\text { Preventive measures against COV-19 in the } \\
\text { dental clinics of Al-Kafeel University. }\end{array}$ \\
\hline & - Notices of restoring dental services. & Working hours of dental emergency clinics \\
\hline $\begin{array}{l}\text { Needs of dental } \\
\text { treatment domain }\end{array}$ & $\begin{array}{l}\text { The public needs of dental services during the epidemic, such as } \\
\text { toothache, orthodontic problem, etc. }\end{array}$ & $\begin{array}{l}\text { I have a dental appointment and I am confused } \\
\text { should I go \# Corona }\end{array}$ \\
\hline \multirow[t]{3}{*}{ Home oral care domain } & $\begin{array}{l}\text { Daily oral care tips, including some commercial ads for oral hygiene } \\
\text { products. }\end{array}$ & Best 5 kinds of mouthwash \\
\hline & - Management of Dental emergencies at home. & How to deal with toothache at home \\
\hline & $\begin{array}{l}\text { - Online consultation services provided by dentists, dental clinics/ } \\
\text { hospitals, online health platforms, etc. }\end{array}$ & $\begin{array}{l}\text { You can contact Dr. lyad in the event of dental } \\
\text { emergency \#COVIDI9 }\end{array}$ \\
\hline
\end{tabular}

July 6, 2020 (Figure 2). Similarly, as seen in Figure 3, the daily number of deaths of COVID-19 too was low during the first 14 days, then increased gradually with fluctuations starting after mid-March, 2020 to May 22, 2020. However, it presented a surge from May 25, 2020, and kept a steady increase with major peaks from mid-June to early-July, and finally presented a major drop on July 6, 2020. Overall, there was no obvious association between time distribution of the tweets to the distribution of new COVID-19 cases and deaths during the study time period from March 2, 2020 to July 6, 2020.

The top five disseminated information frequently retweeted on Twitter and the top five disseminated information frequently liked on Twitter were selected for evaluating the public reactions (Tables 2 and 3 ). The most retweeted information was the help with urgent consultation or emergency dental treatment during COVID-19 tweeted by a dentist. There were 673 retweets and 1,116 likes of this tweet. The second most tweeted information was death of a dentist due to COVID-19 with 523 retweets. The next top three tweets were information of dental treatment cost during COVID-19 pandemic, prevention measures for the coronavirus, and infection of dental school dean with coronavirus. Among the top five liked tweets were a tweet on precautions against the spread of COVID19 among dental students and a tweet on a dentist treating infected COVID-19 patients courageously.

The distributions of tweets containing information about needs of dental treatment in Table 4 show that the most common were complaints of toothaches or wisdom tooth problems, followed by unspecified dental needs, restorative treatment, orthodontic treatment, and implants or prosthetics. Other 


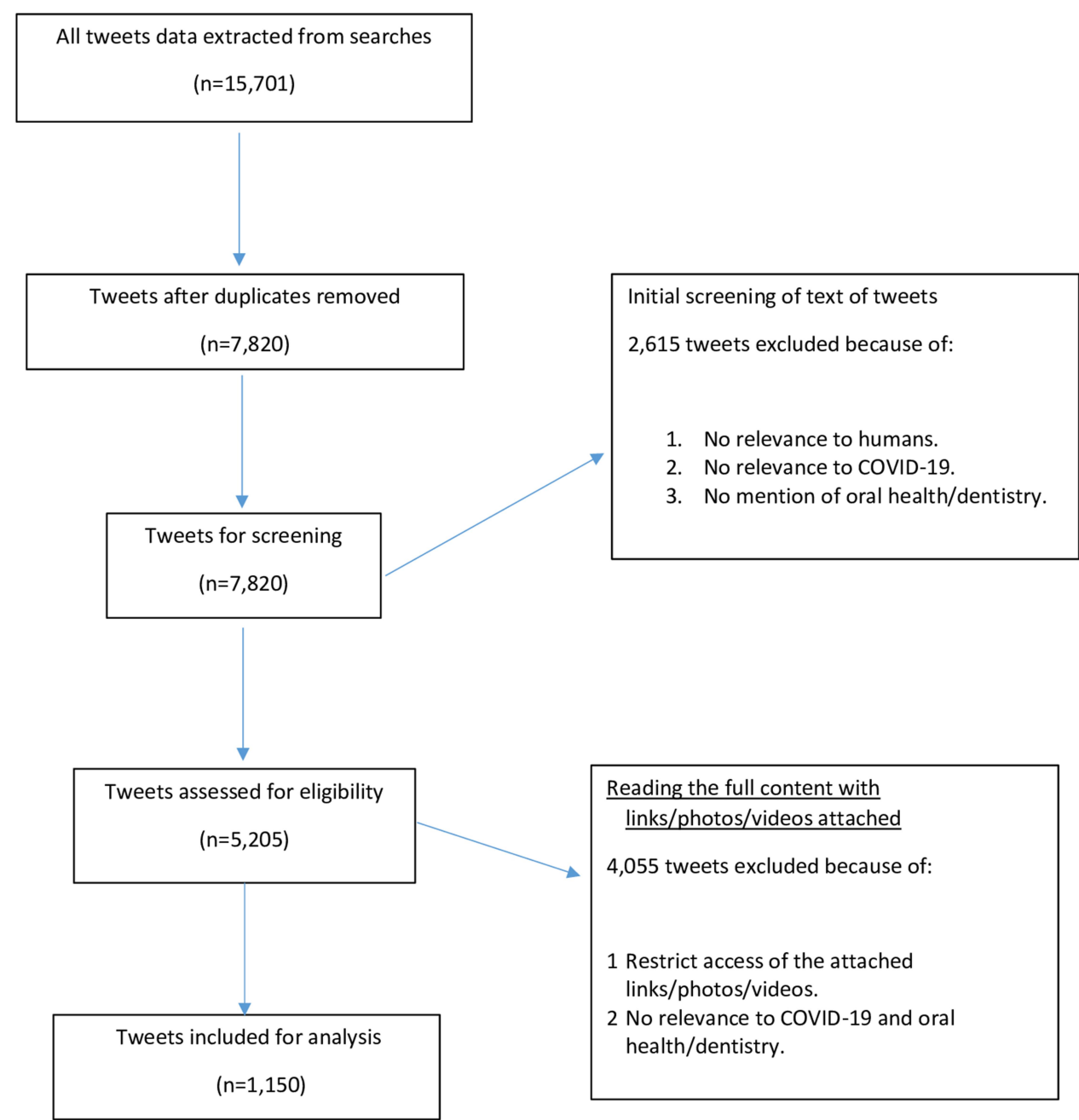

Figure I Flow chart of the study.

treatments were less needed such as Endodontic treatment, Pediatric treatment, Periodontal treatment, and Oral surgery.

Figure 4 shows time distributions of oral health-related tweets during the COVID-19 pandemic. There was a rise in tweets related to the risk of COVID-19 spread during dental procedures during the early stages of the pandemic. There was a noticeable peak of tweets associated with news about stopping all or part of dental services on April 30, 2020 and protective measures on May 4, 2020. When notices of restoring dental services were available from the end of May, there were a stable number of tweets on need for dental treatment during this time.

\section{Discussion}

The aim of this study was to investigate the nature and dissemination of COVID-19 information related to dentistry and oral health information on Twitter platform Arabic database and provide a summary on the supply and demand of dental services during the COVID-19 pandemic on Twitter social media platform. This study is considered a first look at the nature of tweets related to COVID-19 and dentistry with Arab users.

The results of the time distribution of the tweets in this study corroborates with the study done by Mollema et al in 2013, where they also observed a significant drop in the social media attention as the reported number of cases of measles continued to remain high. ${ }^{14}$ The initial peak of tweets in our study can be related to anticipated public concern and interest in learning more about the nature of this novel disease as well as preventive and treatment measures. Similar trend of increased twitter activities level has been reported during the H1N1 outbreak, which corresponded to increased incidence rates. ${ }^{22}$ In addition, there has been a noticeable interest in improving health 


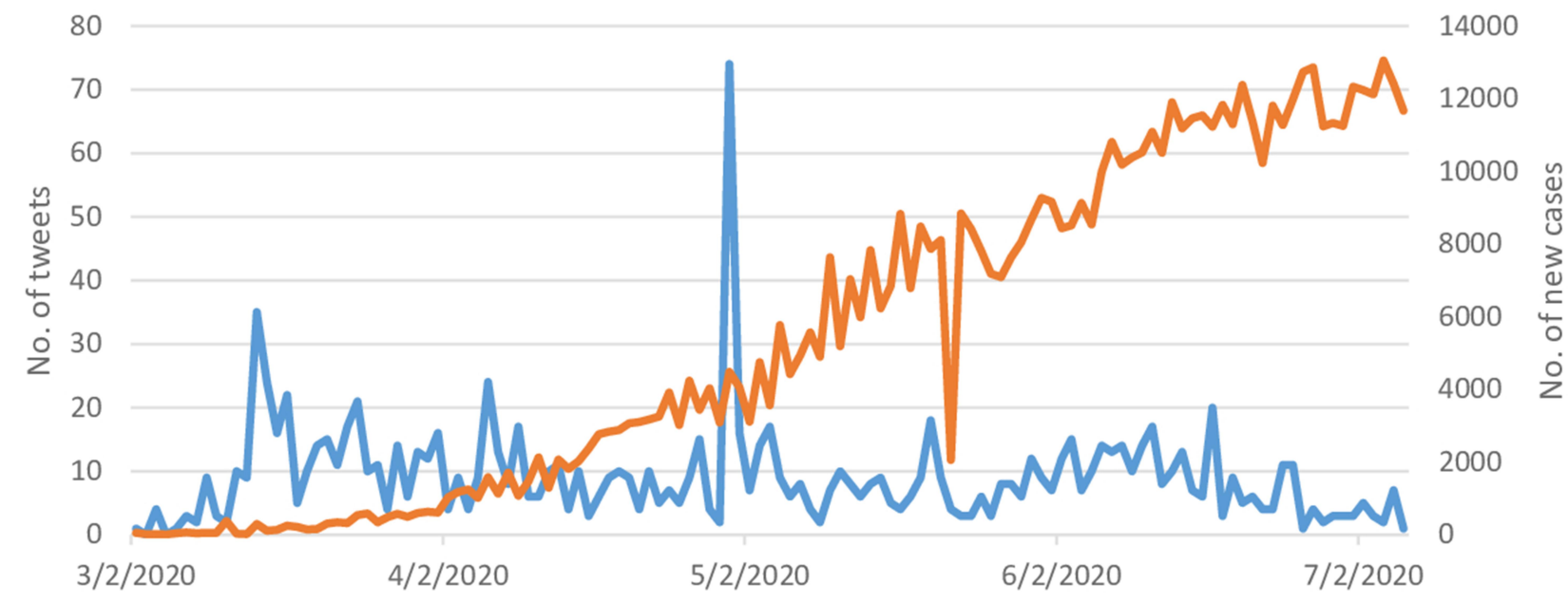

No. of tweets COVID-19 cases

Figure 2 Time distribution of tweets and COVID-19 new cases in the Arab countries.

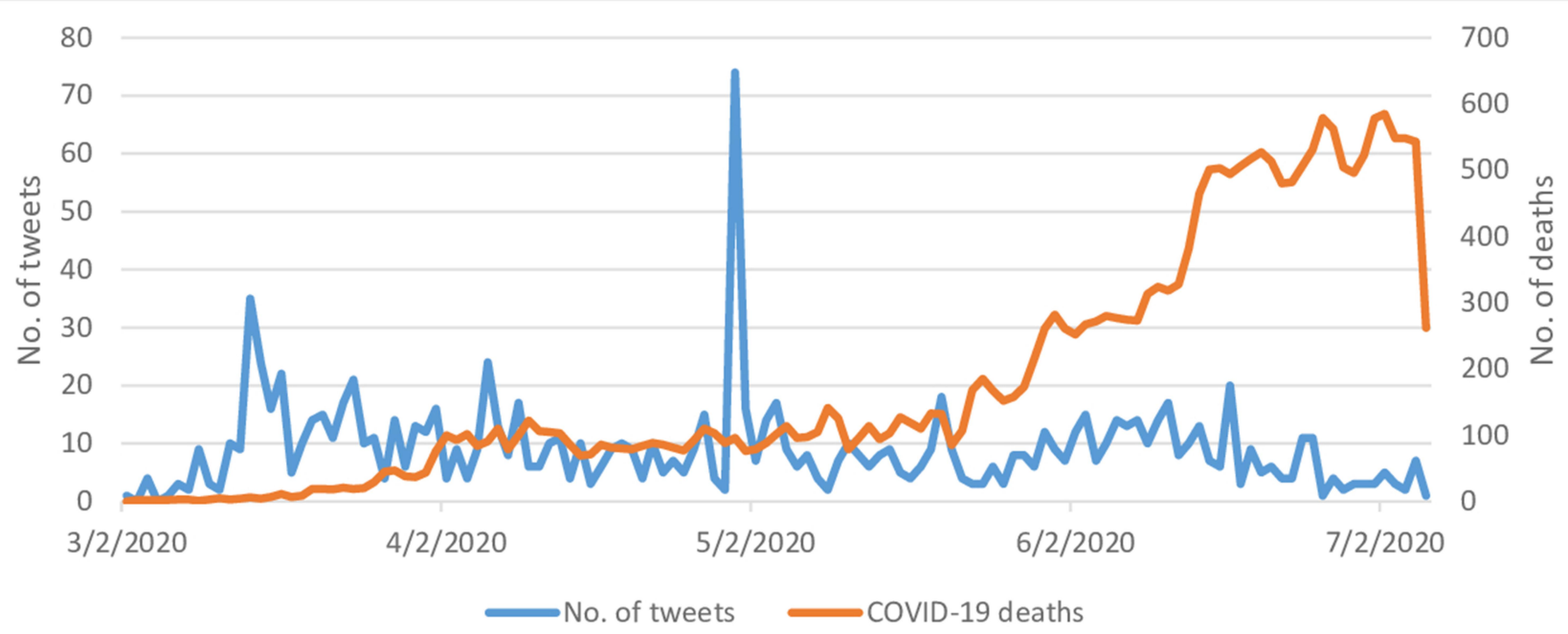

Figure 3 Time distribution of tweets and COVID-19 deaths in the Arab countries.

literacy about protective and preventive measures among Twitter users. ${ }^{23}$ The latter drop in number of tweets, while COVID-19 incidence rate was increasing, can be related to misinformation and dissemination of rumors about COVID-19 from unofficial sources and some social media influencers. Lack of accuracy, bias, and misinformation have been reported to weaken health literacy and induce distrust in information sources. ${ }^{24-26}$ In addition, the drop in number of tweets coincided with the launch of the Saudi Ministry of Health daily COVID-19 reporting on its official Twitter accounts as well as the raise in public awareness about soliciting and sharing information from trustworthy sources. ${ }^{24,27}$
Further analysis of the tweets showed that social media attention gained peak with news about partial or complete closure of dental services on April 30, 2020 and protective measures on May 4, 2020. A steady number of tweets was noticed when information about restoring dental services was available from the end of May. The peak gain emphasizes uncertainty in two issues health literacy and health/risk communication. ${ }^{25,28}$ The effect of limited health literacy became magnified during the pandemic. ${ }^{25}$ Limited oral health literacy regarding oral care has been linked to lack or less than optimal routine dental visits. ${ }^{29}$ On the other hand, the limited Arabic oral health information on governmental sources and official 
Table 2 Top 5 Retweeted Topics During COVID-19

\begin{tabular}{|l|c|}
\hline Top 5 Retweeted Topics & Number of Retweets \\
\hline Help with urgent consultation or emergency dental treatment during COVID-19 tweeted by a dentist & 673 \\
Death of a dentist due to COVID-19 & 523 \\
Dental treatment cost during COVID-19 & 382 \\
Controls and measures for dental clinics' operating routine during COVID-19. & 345 \\
Infection of a Dental school dean with COVID-19 & 114 \\
\hline
\end{tabular}

Table 3 Top 5 Liked Topics During COVID-19

\begin{tabular}{|c|c|}
\hline Top 5 Liked Topics & Number of Likes \\
\hline Help with urgent consultation or emergency dental treatment during COVID-19 tweeted by a dentist & 1,116 \\
\hline Dental treatment cost during COVID-19 & 591 \\
\hline Controls and measures for dental clinics' operating routine during COVID-19. & 462 \\
\hline Precautions against the spread of COVID-19 among dental students & 441 \\
\hline Dentist treating infected COVID-I9 patients courageously & 206 \\
\hline
\end{tabular}

dental organization could have led to such surge in tweeting trend. ${ }^{28}$

Information frequently retweeted on Twitter was analyzed to evaluate public reactions. An earlier study report that virtual platforms and social media are preferred by patients to seek information and share their experiences with peers to get advice, support and reassurance. ${ }^{19,30}$ Analysis of the most frequent retweets gives an insight into the kind of information solicited using the Twitter platform. Analysis of the five most retweeted information showed that the most common was the help with urgent consultation or emergency oral care during COVID-19 tweeted by a dentist, a tweet that was retweeted 673 times and gained 1,116 likes. The need for soliciting oral health reflects the oral health professionals underestimating health literacy needs in such circumstances. ${ }^{25}$ Furthermore, the limited or lack of patient/lay people

Table 4 Needs for Dental Treatment During COVID-I $9(n=462)$

\begin{tabular}{|l|c|}
\hline Needs of Dental Treatment & Number of Tweets n (\%) \\
\hline Restorative treatment & $30(6.49)$ \\
Toothache or wisdom tooth problem & $22 \mathrm{I}(47.83)$ \\
Endodontic treatment & $8(\mathrm{I} .73)$ \\
Oral surgery & $6(\mathrm{I} .29)$ \\
Periodontal treatment & $7(\mathrm{I} .5 \mathrm{I})$ \\
Implants or prosthetics & $\mathrm{II}(2.38)$ \\
Orthodontic treatment & $17(3.68)$ \\
Pediatric treatment & $8(\mathrm{I} .73)$ \\
Unspecified & $154(33.33)$ \\
\hline
\end{tabular}

Arabic information on the professional oral health organization Twitter accounts and websites might have contributed to such information soliciting behavior. ${ }^{28}$ The second and fifth most tweeted information was death of a dentist due to COVID-19 with 523 retweets and 4,019 likes of this news and infection of dental school dean with coronavirus with 114 tweets and 352 likes. News as such gets increased attention on social media due to various sentiments or frustration experienced by the users. ${ }^{12,14}$ The next top two tweets were information of dental treatment cost during COVID-19 pandemic and prevention measures for the coronavirus. Twitter is reported to be an efficient social media tool in spreading information related to health education. ${ }^{31}$

Further analysis of the tweets related to the dental treatment need during COVID-19 showed that $48 \%$ of the tweet was related to toothaches or wisdom tooth problems. This was followed by $33 \%$ of twitter users seeking oral care for dental issues without specific reasons; $6.49 \%$ seeking restorative treatment and fewer than these seeking other specialized dental treatments. Similar finding was reported in earlier studies where the respondents with toothache were more liable to use Twitter to discuss or seek treatment options. ${ }^{32,33}$

An overall analysis of the frequency of tweets related to oral health information exchanged was done to ascertain the demand for dental services during the COVID-19 pandemic. Out of the 1,150 tweets analyzed, $40 \%$ tweets were related to need for dental treatment, followed by $25 \%$ tweets seeking information related to dental services during COVID-19 pandemic and $13 \%$ seeking home oral care information. This 


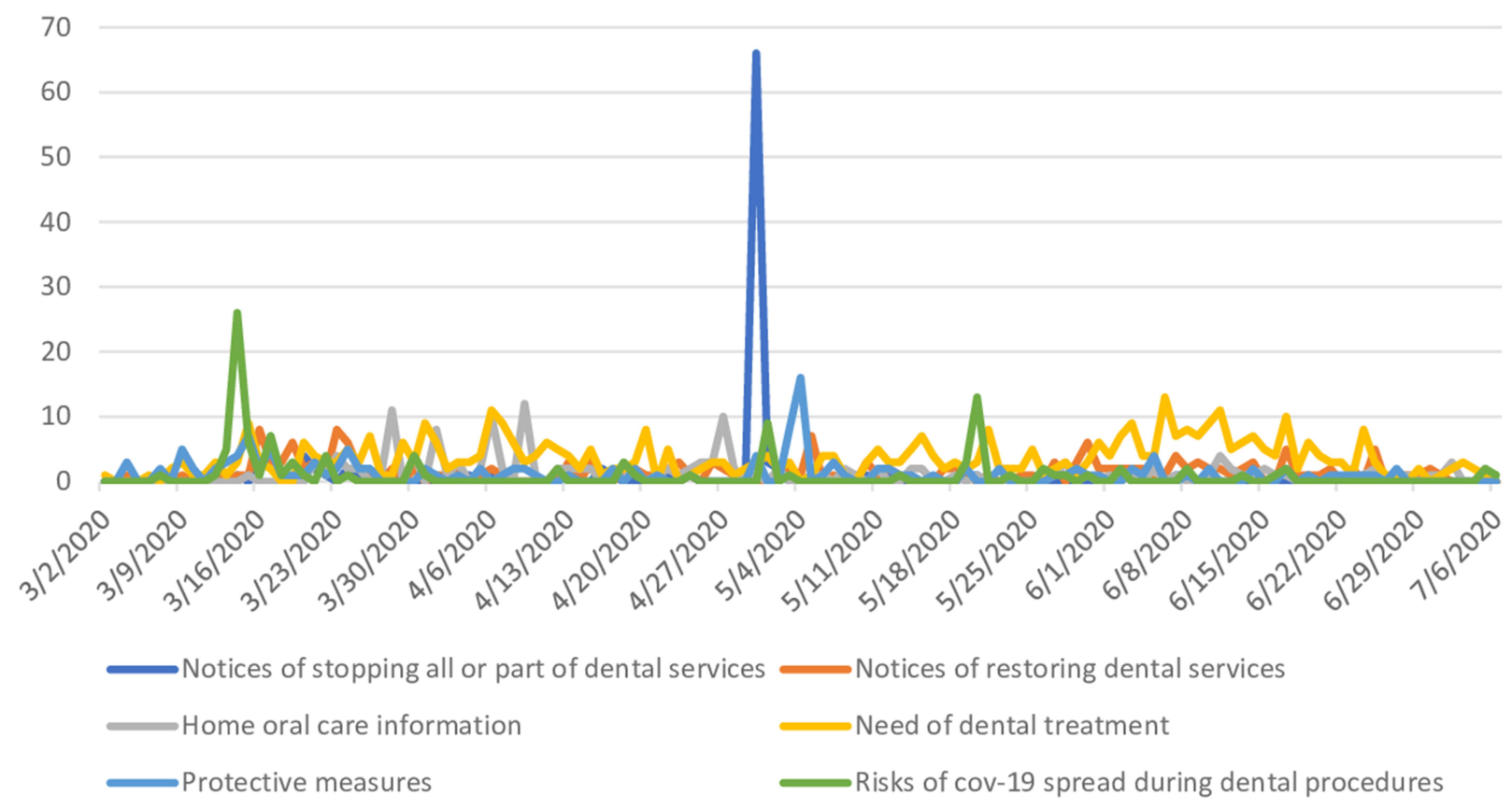

Figure 4 Time distributions of oral health-related tweets during the COVID-19 pandemic.

finding is in agreement with a study done by Heaivilin et al in 2011 where they conclude the use of Twitter as an important health education tool for dental professionals. ${ }^{32}$

Further analysis of the home oral care information tweets revealed that $57 \%$ of the Twitter users were seeking advice from dentists and $38 \%$ of these were particularly interested in seeking information about the daily oral care. Seeking oral health home care information has been associated with lower oral health literacy and less frequent follow-up oral health visits. ${ }^{29,34}$ Only a small proportion of tweets mentioned seeking dental consultation (4.5\%) which could be due to non-availability of dental services during the pandemic.

Thus, the analysis of the nature and dissemination of COVID-19 information related to dentistry on Twitter provides a broad picture of the supply and demand of the dental services during the COVID-19 pandemic. Some of the limitations of this study include the lack of geo-location information of the extracted tweets due to which inferential analysis could not be performed as certain variables such as geo-location and twitter accounts information in addition to other variables were not available. Another limitation was the huge amount of spam found in Arabic social network content. The Arabic language is rich with similar terms for both
COVID-19 and oral health terms which makes it difficult to cover all possible terms. Future studies should ensure using geo-location and twitter account information in addition to using photo and video extraction for the purpose of generalization.

\section{Conclusion}

Based on the results of this study, it is obvious that social media users reacted to the COVID-19 threat to dental practices. Twitter as one of the social media platforms served as a connection between dental health professionals and patients. A comprehensive overview of the tweets related to dentistry and oral health information in the Arabic database on the Twitter platform over a fivemonth period could be used to reflect on the demand and supply of dental services during a pandemic. This study provides insights for policy-makers as well as dental health-care providers both in governmental and private sectors to better enforce online communication and properly utilize social media when routine dental services are unavailable during hostile times such as with the COVID19 pandemic.

\section{Disclosure}

The authors report no conflicts of interest in this work. 


\section{References}

1. Tao ZY, Chu G, McGrath C, et al. Nature and Diffusion of COVID-19-related oral health information on Chinese social media: analysis of tweets on Weibo. J Med Internet Res. 2020;22(6):e19981. doi:10.2196/19981

2. Park HW, Park S, Chong M. Conversations and medical news frames on Twitter: infodemiological Study on COVID-19 in South Korea J Med Internet Res. 2020;22(5):e18897. doi:10.2196/18897

3. European Centre for Disease Prevention and Control. COVID-19 situation update worldwide, as of 8 October 2020. Available from: https://www.ecdc.europa.eu/en/geographical-distribution-2019-ncovcases. Accessed May 8, 2021.

4. Izzetti R, Nisi M, Gabriele M, Graziani F. COVID-19 transmission in dental practice: brief review of preventive measures in Italy. $J$ Dent Res. 2020;99(9):1030-1038. doi:10.1177/0022034520920580

5. Programme UNDP. Monitor the COVID-19 in the Arab States 2020. Available from: https://www.arabstates.undp.org/content/rbas/en/ home/coronavirus.html\#covid19dashboard. Accessed May 8, 2021.

6. Ather A, Patel B, Ruparel NB, Diogenes A, Hargreaves KM. Coronavirus disease 19 (COVID-19): implications for clinical dental care. J Endod. 2020;46(5):584-595. doi:10.1016/j.joen.2020.03.008

7. Lewnard JA, Lo NC. Scientific and ethical basis for social-distancing interventions against COVID-19. Lancet Infect Dis. 2020;20 (6):631-633. doi:10.1016/S1473-3099(20)30190-0

8. Wilder-Smith A, Freedman DO. Isolation, quarantine, social distancing and community containment: pivotal role for old-style public health measures in the novel coronavirus $(2019-\mathrm{nCoV})$ outbreak $J$ Travel Med. 2020;27(2):taaa020. doi:10.1093/jtm/taaa020

9. Al-Khalifa KS, AlSheikh R, Al-Swuailem AS, et al. Pandemic preparedness of dentists against coronavirus disease: a Saudi Arabian experience. PLoS One. 2020;15(8):e237630. doi:10.1371/journal. pone. 0237630

10. Odeh ND, Babkair H, Abu-Hammad S, Borzangy S, Abu-Hammad A, Abu-Hammad O. COVID-19: present and future challenges for dental practice. Int J Environ Res Public Health. 2020;17(9):3151. doi:10.3390/ijerph17093151

11. Karasneh R, Al-Azzam S, Muflih S, Soudah O, Hawamdeh S, Khader Y. Media's effect on shaping knowledge, awareness risk perceptions and communication practices of pandemic COVID-19 among pharmacists. Res Social Adm Pharm. 2020;17(1):SS1551-7. doi:10.1016/j.sapharm.2020.04.027

12. Abd-Alrazaq A, Alhuwail D, Househ M, Hamdi M, Shah Z. Top concerns of tweeters during the COVID-19 pandemic: infoveillance Study. J Med Internet Res. 2020;22(4):e19016. doi:10.2196/19016

13. Hawkins CM, Duszak R, Rawson JV. Social media in radiology: early trends in Twitter microblogging at radiology's largest international meeting. J Am Coll Radiol. 2014;11(4):387-390. doi:10.1016/j. jacr.2013.07.015

14. Mollema L, Harmsen IA, Broekhuizen E, et al. Disease detection or public opinion reflection? Content analysis of tweets, other social media, and online newspapers during the measles outbreak in The Netherlands in 2013. J Med Internet Res. 2015;17(5):e128. doi:10.2196/jmir.3863

15. Singh L, Bansal S, Bode L, et al. A first look at COVID-19 information and misinformation sharing on Twitter. ArXiv. 2020; arXiv:2003.13907v1.

16. Alqurashi S, Alhindi A, Alanazi E. Large Arabic twitter dataset on covid-19. ArXiv. 2020; arXiv:2004.043152.

17. Clement J; Statista. 2020 Oct 8. Leading countries based on number of Twitter users as of October 2020 (in millions). Available from: https://www.statista.com/statistics/242606/number-of-active-twitterusers-in-selected-countries/. Accessed October 8, 2020.
18. Haouari F, Hasanain M, Suwaileh R, Elsayed T. Arcov-19: the first Arabic covid-19 twitter dataset with propagation networks. ArXiv. 2020;arXiv:2004.05861.

19. Donelle L, Booth RG. Health tweets: an exploration of health promotion on twitter. Online J Issues Nurs. 2012;17(3):4.

20. GetOldTweets3. Available from: https://pypi.org/project/ GetOldTweets3/. Accessed July 6, 2020.

21. WHO coronavirus disease (COVID-19) Dashboard. Available from: https://covid19.who.int/. Accessed July 6, 2020.

22. Signorini A, Segre AM, Polgreen PM. The use of Twitter to track levels of disease activity and public concern in the US during the influenza A H1N1 pandemic. PLoS One. 2011;6(5):e19467. doi:10.1371/journal.pone.0019467

23. Winters M, Jalloh MF, Sengeh P, et al. Risk communication and Ebola-specific knowledge and behavior during 2014-2015 outbreak, Sierra Leone. Emerg Infect Dis. 2018;24(2):336-344. doi:10.3201/ eid2402.171028

24. Ramez K, Abi JJ, Afif K, et al. Coronavirus goes viral: quantifying the COVID-19 misinformation epidemic on Twitter. Cureus. 2020;12 (3). doi:10.7759/cureus. 7255

25. Paakkari L, Okan O. COVID-19: health literacy is an underestimated problem. Lancet Public Health. 2020;5(5):e249-e250. doi:10.1016/ S2468-2667(20)30086-4

26. Tasnim S, Hossain MM, Mazumder H. Impact of rumors or misinformation on coronavirus disease (COVID-19) in social media. J Prev Med Public Health. 2020;53(3):171-174. doi:10.3961/jpmph.20.094

27. Mccallum ML, Bury GW. Google search patterns suggest declining interest in the environment. Biodivers Conserv. 2013;22(6-7):1355-1367. doi:10.1007/s10531-013-0476-6

28. Mishra V, Dexter JP. Comparison of readability of official public health information about COVID-19 on websites of international agencies and the governments of 15 Countries. JAMA Netw Open. 2020;3(8):e2018033. doi:10.1001/jamanetworkopen.2020.18033

29. Sabbahi DA, Lawrence HP, Limeback H, Rootman I. Development and evaluation of an oral health literacy instrument for adults. Community Dent Oral Epidemiol. 2009;37(5):451-462. doi:10.1111/ j.1600-0528.2009.00490.x

30. Watts GD, Christou P, Antonarakis GS. Experiences of individuals concerning combined orthodontic and orthognathic surgical treatment: a qualitative Twitter analysis. Med Princ Pract. 2018;27 (3):227-235. doi:10.1159/000487904

31. El Tantawi M, Al-Ansari A, AlSubaie A, Fathy A, Aly NM, Mohamed AS. Reach of messages in a dental twitter network: cohort study examining user popularity, communication pattern, and network structure. J Med Internet Res. 2018;20(9):e10781. doi: $10.2196 / 10781$

32. Heaivilin N, Gerbert B, Page JE, Gibbs JL. Public health surveillance of dental pain via Twitter. J Dent Res. 2011;90(9):1047-1051. doi: $10.1177 / 0022034511415273$

33. Ahlwardt K, Heaivilin N, Gibbs J, Page J, Gerbert B, Tsoh JY. Tweeting about pain: comparing self-reported toothache experiences with those of backaches, earaches and headaches. J Am Dent Assoc. 2014;145(7):737-743. doi:10.14219/jada.2014.30

34. Baskaradoss JK. The association between oral health literacy and missed dental appointments. $J$ Am Dent Assoc. 2016;147 (11):867-874. doi:10.1016/j.adaj.2016.05.011 


\section{Publish your work in this journal}

Risk Management and Healthcare Policy is an international, peerreviewed, open access journal focusing on all aspects of public health, policy, and preventative measures to promote good health and improve morbidity and mortality in the population. The journal welcomes submitted papers covering original research, basic science, clinical \& epidemiological studies, reviews and evaluations, guidelines, expert opinion and commentary, case reports and extended reports. The manuscript management system is completely online and includes a very quick and fair peer-review system, which is all easy to use. Visit http://www.dovepress.com/testimonials.php to read real quotes from published authors.

Submit your manuscript here: https://www.dovepress.com/risk-management-and-healthcare-policy-journal 